\title{
Right Ventricular Endomyocardial Fibrosis in Neonate-A Case Report
}

\author{
Ramachandran Muthiah \\ Thoothukudi Medical College Hospital, Thoothukudi, India \\ Email: cardioramachandran@yahoo.co.uk
}

Received 8 April 2016; accepted 24 May 2016; published 27 May 2016

Copyright (C) 2016 by author and Scientific Research Publishing Inc.

This work is licensed under the Creative Commons Attribution International License (CC BY).

http://creativecommons.org/licenses/by/4.0/

(c) (i) Open Access

\begin{abstract}
Endomyocardial fibrosis is characterized by the formation of fibrous tissue in the endocardium. Although the disease may affect both ventricles, isolated or not, the right ventricle is more frequently involved. Endocardial fibrosis may be present in the entire ventricular cavity, being usually more marked in the apical region and right ventricular inflow tract. Its etiology, however, still remains unknown. Background-Reports on endomyocardial fibrosis in pediatric patients are not common. It is more frequent from the fourth year of age on, with few reports in the first 2 years of life. This case aimed at reporting right ventricular endomyocardial fibrosis in $\mathbf{7}$ days old male neonate, in addition to discussing the etiopathogenesis and future perspective of the disease.
\end{abstract}

\section{Keywords}

Apical Fibrosis, Right Ventricle, Tricuspid Regurgitation, Echocardiography, Neonate

\section{Introduction}

Endomyocardial fibrosis (EMF) is principally a disease of children and young adults in Africa and other tropical areas, characterized by fibrosis of the ventricular endocardium and sub-endocardium that extends from the apex upwards, often involving the tricuspid and mitral valves. The fibrous tissue markedly diminishes the volume and compliance of affected chambers and so induces a restrictive functional defect. The etiology is unknown [1]. Several hypotheses linking infectious agents, toxins, or environmental factors to EMF's unique geographical distribution are yet to be corroborated [2] [3]. According to previously published reports, the disease affects both ventricles in $50 \%-55 \%$ of patients, the Left Ventricle (LV) only in $17 \%-40 \%$ of patients, and exclusively the Right Ventricle (RV) in the remaining 10\% - 28\% [4].

The incidence of EMF is highest in Sub-Saharan Africa and regional variations in distribution have been reported in other countries in tropical and subtropical regions. It has been reported in Nigeria, Uganda, Kenya, 
Tanzania, Mozambique, Gabon, Congo, Cameroon, Sudan, Cote d'Ivoire, Ghana, Brazil and India. Initial report from Nigeria was of the opinion that it occurs in hot and humid coastal areas [5]. The majority of EMF cases have come from low-lying, humid parts of tropical countries. In East Africa, Uganda has a striking burden of EMF. In Tanzania and Mozambique, cases have clustered along the coastal forests. In India, Kerala's tropical rain forest has generated one of the largest case series in the world, while other parts of the country have reported relatively few cases. In China, the largest number of case reports has also come from the Southern province of Guangxi [6]. In South America, patients with EMF have come from Brazil and Columbia.

\section{Review of Literature}

The basic lesion of EMF is ventricular endocardial fibrosis. In fact, in 1938, Arthur Williams, the foundation professor of medicine at Mekerere University, Kampala, Uganda, had described two cases of mitral incompetence and correlated with large patches of fibrosis affecting the ventricular walls at necropsy and this is perhaps the earliest documentation of EMF in literature. The pathologist Jack NP Davies first coined the term endomyocardial fibrosis (EMF) in Uganda and described the classical four pathological features and its distribution in Africa [7] at the Royal Society Meeting in 1954 and the disease came to be known as Davies' disease. The typical features described were right and left ventricular endocardial fibrosis, affecting the apex and inflow region with atrioventricular valve regurgitation. At the Mulagu hospital in Uganda, where he worked, the disease accounted for $15 \%$ of the deaths due to heart failure [8]. Bedford, who visited the university in 1948, encouraged him to publish his observations and Arthur Williams and JD Ball were his co-workers. Later JD Ball, with his missionary job joined Christian Medical College, Vellore in India and identified pathological specimens at autopsy which he shipped to Davies for confirmation. Thus the disease was reported from India for the first time.

Right ventricular endomyocardial fibrosis in neonate is uncommon and so this case had been reported.

\section{Case Report}

A 7 days old male child was referred from neonatal intensive care unit for echocardiographic evaluation. A history of an episode of febrile illness affected the mother for one week duration during the $5^{\text {th }}$ month of gestation of this first child. The child was dyspneic and the pulse rate was $124 \mathrm{bpm}$. Physical examination revealed bilateral rhonchi in the lung fields suggesting wheezing bronchitis. Cardiac examination revealed no abnormal findings clinically. Blood chemistry, X-ray chest and ECG were normal. The child was treated with antibiotics and bronchodilators and advised periodic follow up. Screening of family members revealed normal.

Transthoracic 2D echocardiographic images of the child revealed right ventricular apical fibrosis extending to the inflow of the tricuspid valve as shown in Figure 1, mild tricuspid regurgitation as in Figure 2 and Figure 3. The right ventricular outflow tract and pulmonary artery are normal as shown in Figure 4.

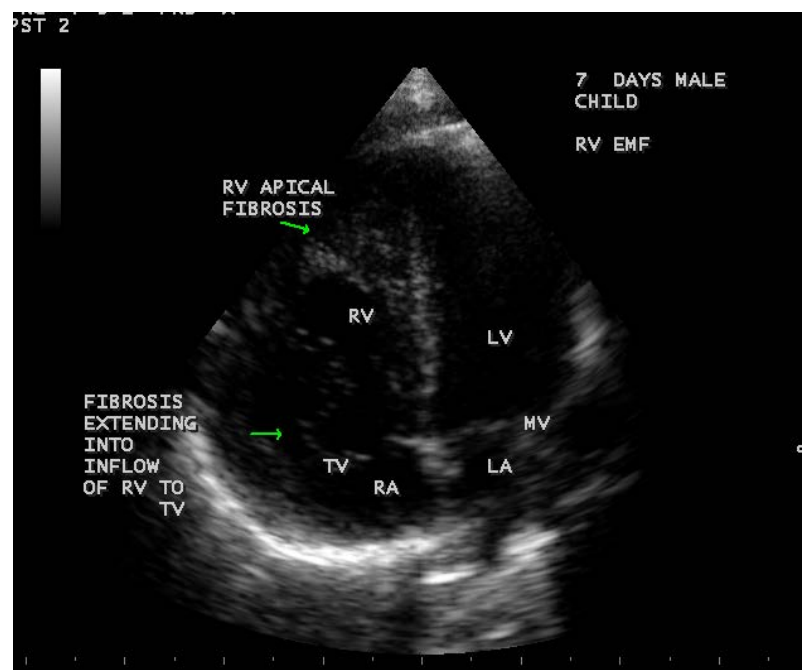

Figure 1. Apical 4 chamber view illustrates the apical fibrosis of right ventricle and its extension to tricuspid valve. 


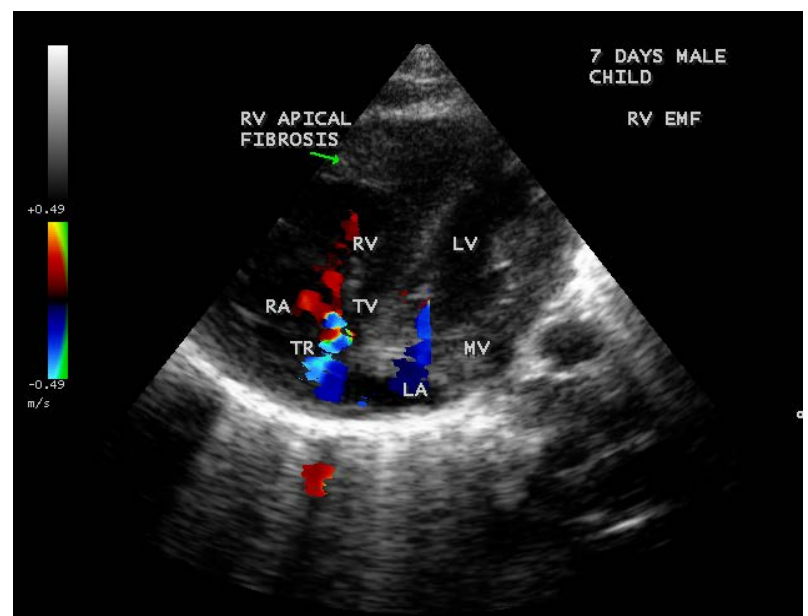

Figure 2. Tilted apical 4 chamber view illustrates the right ventricular apical fibrosis and mild tricuspid regurgitation.

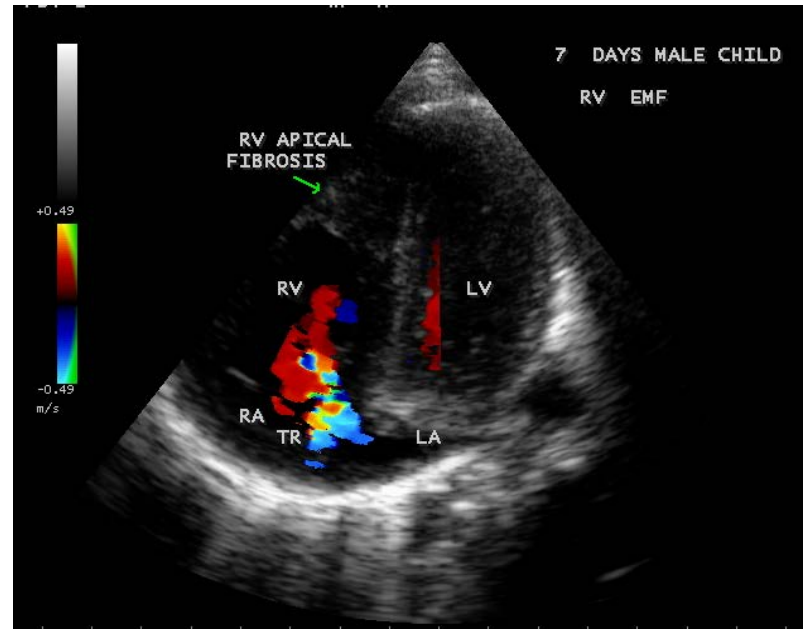

Figure 3. Apical 4 chamber view illustrates the obliteration of right ventricular apex by fibrosis and mild tricuspid regurgitation.

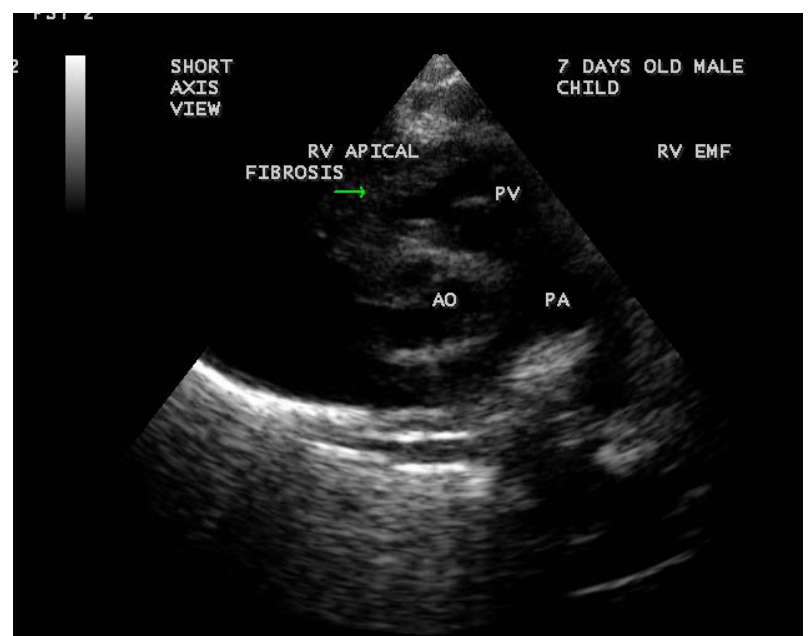

Figure 4. Short axis view showing the normal RV outflow tract, pulmonary valve and pulmonary artery. 


\section{Discussion}

\subsection{Age Distribution}

The frequency of EMF cases in Uganda has a bimodal peak at age 10 and age 30 [9]. Childhood EMF in this country affects boys and girls equally, while adult EMF affects woman twice as often as men [10]. In Nigeria, some studies have found a two to one male preponderance, while others have not shown any difference between sexes. Majority of cases in India from Kerala state are below the age of 40 years. EMF is not generally observed in children younger than 4 years, although the typical pathology of EMF has recently been described in a 4month-old infant. EMF appears to be more prevalent in certain ethnic groups. One study in Uganda showed that EMF is more common in individuals with Rwanda/Burundi ethnic origins.

\subsection{Etiopathogenesis}

The etio-pathogenesis of EMF remains much of an enigma, with several unvalidated hypothesis ranging from infection, inflammatory and dietary causes as well as eosinophilic toxicity play a role in tropical regions. They include cerium deficiency, magnesium deficiency, cassava, malnutrition, parasitic diseases, viral diseases, autoimmunity, allergy, and ethnicity. The hypotheses postulated to explain the etiology of EMF have not been proven and cannot explain its occurrence worldwide.

Davies himself, who died in 1998 at the age of 83, believed to the end that EMF had a unifying explanation. He thought the clue perhaps lay in the similarity between the heart lesion in EMF and the endocarditis parietalis fibroplastica that Wilhem Loffler and others had described in Europe in the setting of hypereosinophilic syndromes [9]. The eosinophil hypothesis-dominant though still a not well tested-has failed to convince critics who point to other plausible alternatives. In fact, more of the etiologic categories first mentioned by Williams, Ball, and Davies in 1954 have left Table 1 of possible causes given below.

By and large, the endomyocardial fibrosis could be a reaction pattern of the endocardium to a variety of insults [11]. Role of anti-heart antibodies similar to rheumatic process may be involved in the fibrotic process of EMF. The endocardial lesions in EMF are said to evolve in 3 successive stages, namely, necrosis, thrombosis, and fibrosis [12] and these stages have been defined on the basis of postmortem material in Loffler's endocarditis by Olsen. Davies has described three phases of the disease [13]. The initial phase is an acute carditis phase characterized by febrile illness and in severe cases with heart failure and shock, followed by progress into a

Table 1. Proposed causes of endomyocardial fibrosis.

\begin{tabular}{|c|c|}
\hline Etiology & Causes \\
\hline \multirow[t]{5}{*}{ Infection } & Toxoplasmosis \\
\hline & Rheumatic fever \\
\hline & Malaria \\
\hline & Myocarditis \\
\hline & Helminthic parasites \\
\hline \multirow[t]{2}{*}{ Allergy } & Eosinophilia \\
\hline & Auto-immunity \\
\hline \multirow[t]{2}{*}{ Malnutrition } & Protein deficiency \\
\hline & Magnesium deficiency \\
\hline \multirow[t]{6}{*}{ Toxic Agents } & Cerium \\
\hline & Cassava \\
\hline & Thorium \\
\hline & Serotonin \\
\hline & Plant toxin \\
\hline & Vitamin D \\
\hline
\end{tabular}


subacute phase and a chronic phase. Most of the patients come to clinical attention in the chronic burnt-out phase. The early part of the disease is rarely clinically recognized in India and the disease come to attention in the late stages and isolated endocardial involvement and intracardiac thrombi are the peculiar features [14]. The climatic restriction of areas where EMF is endemic and the reports of sporadic cases in foreigners after short visits to these areas suggest a role of infectious agents in the pathogenesis of the disease [15]. Increased levels of interleukin-6, endothelial cell activation, and increased fibrinolysis were found in patients with recent-onset EMF, strongly suggesting that inflammation, endothelial injury, and procoagulant changes are major players in the pathogenesis of this condition [16].

Histology of the heart shows abnormalities in all 3 layers [17]. The most prominent lesion is endocardial thickening, which is due to acellular fibrocollagen tissue deposits under a layer of normal endocardium [18]. While in general, fibrosis in cardiac tissue has been mainly linked to increased level of cytokine, transforming growth factor $\beta_{1}$ [19], the underlying mechanism of myocardial fibrosis in this entity remain unclear.

\subsection{Echocardiography}

Echocardiography is the most valuable tool for the diagnosis of EMF [20]. Mocumbi et al. [14] have described the echocardiographic criteria to diagnose EMF and the criteria described in the literature apply only to the disease in the advanced stages [21]. The presence and location of fibrosis as determined by echocardiography correlate well with autopsy findings. Obliteration of the apex of the involved ventricle is the hallmark of the disease. Ventricular obliteration and cavity retraction are described features of right EMF, clearly depicted on echocardiography. More specifically, EMF is a disease of the inflow tract endocardium, with severe fibrosis extending from the papillary muscles to the apex of the ventricles [22]. Outflow tract is usually preserved. Atrioventricular valves are also usually preserved, but infiltration of the subvalvular apparatus may cause insufficiency. Thickening of the endocardium and infiltration of the myocardium are responsible for partial obliteration of ventricular cavities, resulting in decreased distensibility and diastolic filling. When symptoms of cardiac failure ensue, the prognosis becomes poor.

In this neonate, transthoracic $2 \mathrm{D}$ echocardiographic images showed fibrotic obliteration of the right ventricular apex and its extension into the inflow of the tricuspid valve as shown in Figure 1, with mild tricuspid regurgitation as shown in Figure 2 and Figure 3 and the free RV outflow tract, pulmonary valve and artery as in Figure 4 suggesting the Right ventricular endomyocardial fibrosis

\subsection{Future Perspective}

There is a history of febrile illness for one week duration during the 5th month of gestation in the antenatal period of mother of this child and echocardiography of mother revealed normal. The exact etiology of this febrile illness was unknown. Recent studies have demonstrated antibodies against myocardial proteins and a high incidence of cardiotrophic infectious agents like cytomegalovirus, Epstein-Barr virus and Toxoplasma gondii in affected patients suggesting a role of inflammation [23]. A recent study states that the right ventricle was the most affected cavity in EMF and majority of patients were between 5 and 15 years of age group [24] similar to rheumatic fever.

Echocardiographic screening of family members revealed normal even though they are living with same environmental exposure and dietary habits. Some unknown cardiotrophic infectious agents produce inflammatory mediators, cause immune mediated injury to the apical endocardium and inflow portion of the ventricles, result in fibrotic process obliterating the apex and affect the subvalvular apparatus similar to rheumatic process. Thus, future research must focus on identifying the strains of particular organisms responsible for this enigmatic disease worldwide.

\section{Conclusion}

Right ventricular endomyocardial fibrosis described by 2D echocardiographic images in a 7 days old male neonate is rare in the literature and it is detected in the coastal region of Thoothukudi in Tamil Nadu state at this tropical zone of India.

\section{References}

[1] Schoen, F.J. and Mitchell, R.N. (2010) Robbins and Cotran, Pathologic Basis of Disease. Chap. 12, 8th Edition, 
Saunders, Philadelphia, 577.

[2] Bukhman, G., Ziegler, J. and Parry, E. (2008) Endomyocardial Fibrosis, Still a Mystery after 60 Years. Achieve of Public Library of Science (PLOS) Neglected Tropical Diseases, 2, e97. http://dx.doi.org/10.1371/journal.pntd.0000097

[3] Shaper, A.G. (1970) The Geographical Distribution of Endomyocardial Fibrosis. Indian Journal of Pathology and Microbiology (Basel), 35, 26-35. http://dx.doi.org/10.1159/000162195

[4] Gergana, M.-S., Eivgeny, H., Ami, N., Cecilia, G.M., Dario, C.G. and Alon, S. (2009) Prevalence of Exclusively Right-Sided Endomyocardial Fibrosis among Patients with Heart Failure in Equatorial Guinea. The Open Tropical Medicine Journal, 2, 24-26. http://dx.doi.org/10.2174/1874315300902010024

[5] Falase, A.O. (1983) Endomyocardial Fibrosis in Africa. Postgraduate Medical Journal, 59, 170-178. http://dx.doi.org/10.1136/pgmj.59.689.170

[6] Yin, R. (2000) Endomyocardial Fibrosis in China, Chinese Medical Sciences Journal, 15, 55-60.

[7] Williams, A.W., Ball, J.D. and Davies, J.N. (1954) Endomyocardial Fibrosis in Africa, Its Diagnosis, Distribution and Nature. Transactions of the Royal Society of Tropical Medicine and Hygiene, 48, 290-305. http://dx.doi.org/10.1016/0035-9203(54)90100-5

[8] Connor, D.H., Somers, K., Hutt, M.S., Manion, W.C. and D’Arbela, P.G. (1968) Endomyocardial Fibrosis in Uganda (Davies’ Disease), Part II, an Epidemiologic, Clinical, and Pathologic Study. American Heart Journal, 75, 107-124. http://dx.doi.org/10.1016/0002-8703(68)90122-1

[9] Corssmit, E.P., Trip, M.D. and Durrer, J.D. (1999) Loffler’s Endomyocarditis in the Idiopathic Hypereosinophilic Syndrome. Cardiology, 91, 272-276. http://dx.doi.org/10.1159/000006923

[10] Davies, J., Spry, C.J., Vijayaraghavan, G. and De Souza, J.A. (1983) A Comparison of the Clinical and Cardiological Features of Endomyocardial Disease in Temperate and Tropical Regions. Postgraduate Medical Journal, 59, 179-185. http://dx.doi.org/10.1136/pgmj.59.689.179

[11] Kartha, C.C. (1995) Endomyocardial Fibrosis, a Case for the Tropical Doctor. Cardiovascular Research, 30, 636-643. http://dx.doi.org/10.1016/S0008-6363(96)88509-9

[12] Olsen, E.G.J. and Sekiguchi, M. (1990) Morphological Overview and Pathogenic Mechanisms in Endomyocardial Fibrosis-Associated with Eosinophilia. Cardiomyopathy Update, 3, 21.

[13] Davies, J.N. (1968) The Ridge in Endomyocardial Fibrosis. The Lancet, 1, 631-632. http://dx.doi.org/10.1016/S0140-6736(68)91250-6

[14] Mocumbi, A.O., Ferreira, M.B., Sidi, D. and Yacoub, M.H. (2008) A Population Study of Endomyocardial Fibrosis in a Rural Area of Mozambique. New England Journal of Medicine, 359, 43-49. http://dx.doi.org/10.1056/NEJMoa0708629

[15] Brockington, I.F., Olsen, E.G. and Goodwin, J.F. (1967) Endomyocardial Fibrosis in Europeans, Resident in Tropical Africa. The Lancet, 1, 583-588. http://dx.doi.org/10.1016/S0140-6736(67)90440-0

[16] Mocumbi, A.O. (2008) Epidemiology, Pathogenesis and Management of Endomyocardial Fibrosis. PhD Thesis, Imperial College, London.

[17] Farrer-Brown, G., Tarbit, M.H., Somers, K. and Hutt, M.S.R. (1972) Microvascular Study of Hearts with Endomyocardial Fibrosis. British Heart Journal, 34, 1250-1262. http://dx.doi.org/10.1136/hrt.34.12.1250

[18] Seth, S., Thatai, D., Sharma, S., Chopra, P. and Talwar, K.K. (2004) Clinico-Pathological Evaluation of Restrictive Cardiomyopathy (Endomyocardial Fibrosis and Idiopathic Restrictive Cardiomyopathy) in India. Europeon Journal of Heart Failure, 6, 723-729. http://dx.doi.org/10.1016/j.ejheart.2003.11.009

[19] Sovari, A.A., Morita, N., Weiss, J.N. and Karagueuzian, H.S. (2008) Serum Transforming Growth Factor- $\beta_{1}$ as a Risk Stratifier of Sudden Cardiac Death. Journal of Medical Hypotheses and Ideas, 71, 262-265.

[20] Mocumbi, A. (2009) Neglected Cardiomyopathies in Africa. South African Heart Journal, 6, 30-41.

[21] Berensztein, C.S., Pineiro, D., Marcotegui, M., Brunoldi, R., Blanco, M.V. and Lernan, J. (2000) Usefulness of Echocardiography and Doppler Echocardiography in Endomyocardial Fibrosis. Journal of American Society of Echocardiography, 16, 385-392. http://dx.doi.org/10.1016/S0894-7317(00)70008-3

[22] Davies, J.N. (1969) The African Cardiomyopathies. Annals of the New York Academy of Sciences, 156, 498-503. http://dx.doi.org/10.1111/j.1749-6632.1969.tb16747.x

[23] Iglezias, S.D., Benvenuti, L.A., Calabrese, F., Salemi, V.M., Silva, A.M., Carturan, E., De Oliveira, S.A., Thiene, G. and De Brito, T. (2008) Endomyocardial Fibrosis: Pathological and Molecular Findings of Surgically Resected Ventricular Endomyocardium. Virchows Archiv, 453, 233-241. http://dx.doi.org/10.1007/s00428-008-0652-3

[24] Chelo, D., Nguefack, F., Mbassi Awa, H.D. and Kingue, S. (2016) Endomyocardial Fibrosis in Sub Saharan AfricaThe Geographical Origin, Socioeconomic Status, and Dietary Habits of Cases Reported in Yaounde, Cameroon. Annals of Pediatric Cardiology, 8, 202-209. 